\title{
Slip Behavior and Hardness Indentations in MnSe and MnSe-MnS Solid Solutions
}

\author{
PAUL G. RIEWALD* and LAWRENCE H. VAN VLACK \\ Department of Chemical and Metallurgical Engineering, The University of Michigan, Ann Arbor, Michigan 48104
}

\begin{abstract}
Single crystals in the solid solution series MnSe-MnS were prepared, and selected surfaces were indented with either a Vickers or a Knoop microindenter. Information on mechanical behavior was obtained by observation of slip traces around indentations and by study of Knoop hardness anisotropy. Manganous selenide exhibits $\{111\}<11 \overline{0}>$ and $\{110\}$ $<110>$ as primary and secondary slip mechanisms, respectively, in contrast to the $\{110\}<110\rangle$ mechanism preferred by MnS. In the system MnSe-MnS, the primary slip mechanism changes gradually with composition. The $\{110\}<\overline{110}\rangle$ mechanism of $\mathrm{MnS}$ is much more sensitive to temperature than the $\{111\}<110>$ mechanism of MnSe, and a large solid solution hardening effect accompanies the substitution of sulfide for selenide ions.
\end{abstract}

\section{Introduction and Previous Work}

$T$ His paper presents results of a study on the crystallographic deformation mechanisms, fracture characteristics, and hardness relations for single crystals of the solid solution series MnSe-MnS. Such a study allows comparison of mechanical behavior in two compounds where anions rather than cations are varied.

The MnS and MnSe used in this investigation have the facecentered cubic, NaCl-type structure. Both, however, show two other allotropic modifications ${ }^{1}$ : a cubic zincblende (sphalerite) structure and a hexagonal wurtzite form. At low temperatures, both undergo an antiferromagnetic transformation which results in a slight deformation of the cubic unit cell into one showing rhombohedral symmetry.,3 Sirota and Makovetskii ${ }^{4}$ report that, at $115^{\circ} \mathrm{K}, \mathrm{MnSe}$ is partly transformed into its sphalerite modification.

The system MnSe-IMnS was investigated by Mehta et al. ${ }^{5}$ and by Kiessling et $a .^{6}{ }^{6}$ Both found complete solid solubility for all compositions, although Kiessling et al. determined solid solubilities at $1150^{\circ} \mathrm{C}$ only.

Numerous investigators have studied plastic deformation behavior in a variety of NaCl-type crystals, and Gilman ${ }^{7}$ has prepared a review of the mechanical behavior which indicates that most NaCl-type crystals have primary deformation in $<110\rangle$ directions on $\{110\}$ planes. Secondary glide mechanisms, especially at high temperatures, include $\{100\}<011\rangle$ and $\{111\}<1 \overline{1} 0>$. Lead sulfide and lead telluride, which are highly polarizable, show slip primarily on $\{100\}$ planes. Uranium carbide and titanium carbide, which are largely covalent compounds, have the $\{111\}<1 \overline{1} 0\rangle$ slip mechanism as primary." Some rock salt-type crystals such as $\mathrm{AgCl}$ and

Presented at the Fall Meeting of the Basic Science and Electronics Divisions of The American Ceramic Society, St. Paul, Minn., September 19, 1968 (Paper No. 29-BE-68F). Received October 16,1968; revised copy received February 10, 1969.

Based in part on a thesis submitted by Paul G. Riewald for the Ph.D. degree in metallurgical engineering, The University of Michigan, August 1968.

* Now with the Pioneering Research Laboratory, DuPont Experimental Station, Wilmington, Delaware 19898.
AgBr show little preference for any glide plane; rather, slip appears to take place on any plane for which the slip direction is a zone axis. ${ }^{7}$

The microhardness indentation technique used in this investigation to study slip mechanisms was previously used by $\mathrm{Keh}^{9}$ with $\mathrm{MgO}$ single crystals. Only the $\{110\}<1 \overline{10}>$ slip mechanism was identified. Chao et al., ${ }^{10}$ using the same approach, found that $\{110\}<1 \overrightarrow{1} 0\rangle$ is the primary slip mechanism in $\mathrm{MnS}$, with secondary glide on $\{111\}$ planes.

Fracture in NaCl-type crystals is characterized by easy $\{100\}$ cleavage with secondary cleavage on $\{110\}$ planes. $^{7}$ Keh $^{9}$ and Chao et $a .^{10}$ found $\{110\}$ fracture around Vickers indentations on $\mathrm{MgO}$ and $\mathrm{MnS}$ single crystals and concluded that this was the result of interactions of dislocation movements on adjacent $\{110\}$ slip planes.

\section{Experimental Procedure}

\section{(1) Raw Materials Preparation and Purification}

Manganous sulfide was prepared by the sulfur deoxidation of reagent grade manganous sulfate in an apparatus described by Chao et al. ${ }^{\text {so }}$ A chemical analysis of the green NaCl-type MnS prepared in this way showed $36.8 \pm 0.2 \%$ sulfur compared with $36.9 \%$ from stoichiometry. The $\mathrm{MnS}$ was further purified by a zone refining process. About $60 \mathrm{~g}$ of the unpurified material was placed in a graphite boat and remelted under a purified argon atmosphere within a horizontal moving coil induction furnace. After four or five passes at $3 \mathrm{in} . / \mathrm{h}$, the MnS developed a coarsely crystalline grain size. Microscopic and X-ray examination revealed no other phases, and X-ray data gave a lattice parameter of $5.224 \AA$ compared with $5.224 \AA$ reported by ASTM (Card 6-0518) and 5.225 $\AA$ reported by Chao et $a l^{10}$

Manganous selenide was prepared from manganese metal powder, made from triply vacuum distilled electrolytic manganese, and selenium powder, prepared from high purity selenium shot. The powders were mixed in appropriate proportions, placed in a vacuum degassed graphite crucible, encapsulated under vacuum in a Pyrex tube, and reacted at $600^{\circ} \mathrm{C}$ for several days. The MnSe was also purified by a zone refining process, but the technique was slightly different from that used for $\mathrm{MnS}$. When $\mathrm{MnSe}$ is melted in graphite under argon at 1 atm total pressure, it slowly decomposes with the formation of manganese carbide and elemental selenium. It was necessary, therefore, to seal the graphite crucible containing the MnSe in a fused silica tube thereby allowing a back pressure of Se to be built up, which prevented the reaction of $\mathrm{Mn}$ with the crucible. A two-turn induction coil was used for zone-refining. A secondary furnace was placed around the sample to prevent cracking during cooling of the fused silica tube which had partially crystallized.

Eight to ten passes were made at 3 in. $/ \mathrm{h}$, and the first $60 \%$ of the ingot was saved. Microscopic examination revealed no other phases except for an occasional small inclusion, apparently of $\mathrm{MnO}$. X-ray data gave a lattice parameter of 5.463 compared with $5.462 \AA$ reported by ASTM (Card 11-683). Chemical analysis gave $40.8 \pm 0.4 \% \mathrm{Mn}$ compared with $41.0 \%$ from stoichiometry. 

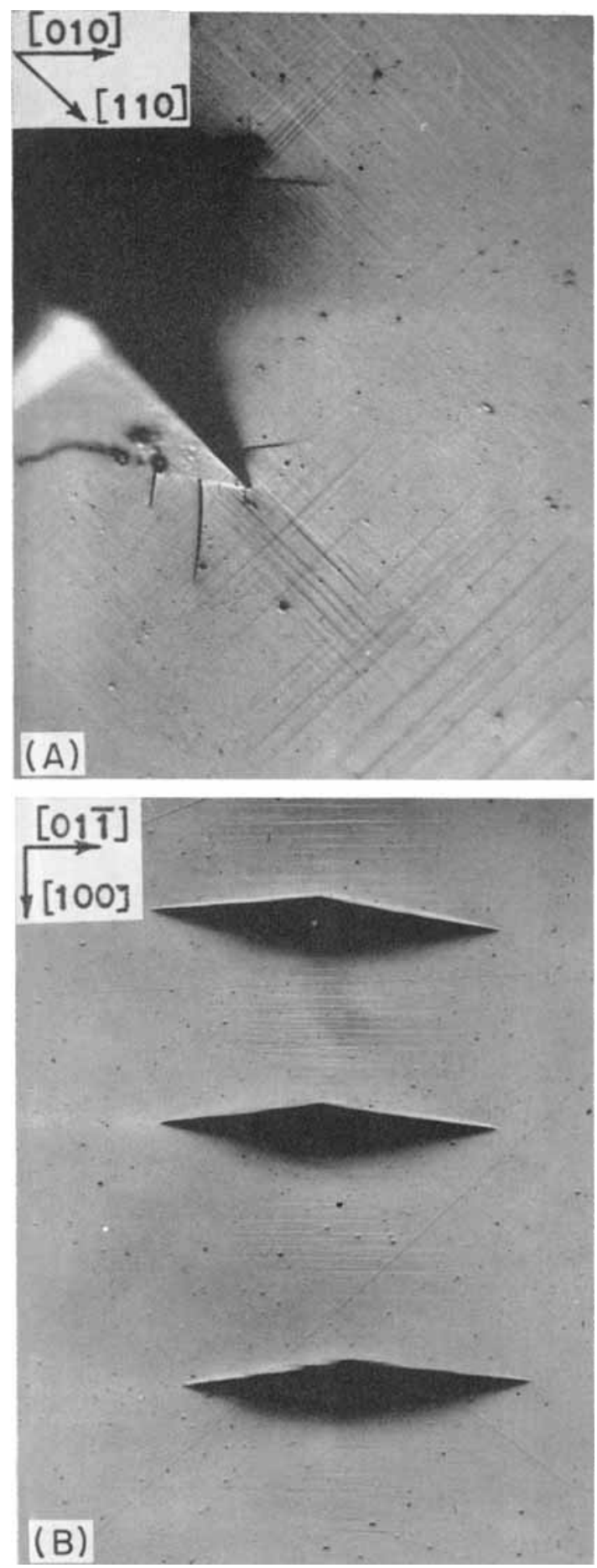

\section{(2) Crystal Preparation}

The zone-refined MnS provided grains large enough to be used in single crystal studies. purified MnSe (about $30 \mathrm{~g}$ ) was placed in a graphite crucible sealed in fused silica. This was placed inside a larger fused silica tube which was lowered slowly through an induction coil to produce single crystals large enough for study. To prepare binary compositions, the individual components were crushed to about 50 mesh, mixed in the desired proportions, and then handled in the same manner as pure MnSe.

Single crystals were cleaved from the large grains previously prepared and annealed $12 \mathrm{~h}$ at $1000^{\circ} \mathrm{C}$. They were then oriented by the Laue back-reflection technique and cut to reveal the crystal surfaces (001), (011), and (111). These orientations were generally accurate to within $2^{\circ}$.

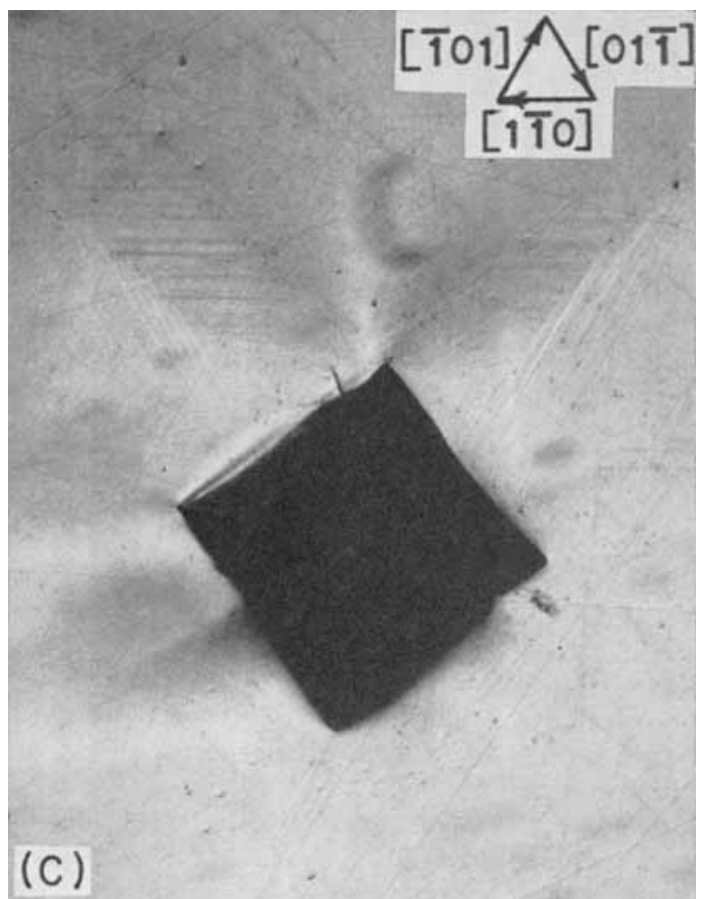

Fig. 1. Vickers and Knoop hardness impressions on the (A) $(001), \times 450 ;(B)(011), \times 250$; and $(C)$ (111), $\times 250$ surfaces of an MnSe crystal showing traces due to $\{111\}<\overline{110}>$ slip.

Samples were mounted in Bakelite (or solder for low temperature testing) and mechanically polished using standard micrographic techniques. Care was taken during the final stages of polishing to remove chemically any possible disturbed surface layers with an etchant of composition $1 \% \mathrm{H}_{2} \mathrm{SO}_{4}$, $1 \% \mathrm{H}_{3} \mathrm{PO}_{4}$, and $1 \%$ of a saturated solution of oxalic acid. Subsequent Laue photographs revealed no significant residual plastic deformation in the crystals.

\section{(3) Testing and Examination}

The mounted and polished samples were then indented with either a Vickers or a Knoop diamond at room temperature, $-196^{\circ},-70^{\circ}$, and $135^{\circ} \mathrm{C}$. The long axis of the Knoop indenter was made parallel to the several low index crystallographic directions on each of the planes tested.

Slip lines were observed directly around the indentations in obliquely reflected light. By noting the orientations of these lines on the several crystal surfaces, the plane or planes on which slip occurred could be deduced. Slip was also studied by observing Knoop hardness variations as a function of indenter orientation and sample composition.

Fracture was studied by inducing cleavage on several of the crystal planes and by noting fracture surfaces in crushed fragments. Also, any fracturing around indentations was related to slip interactions.

\section{Results and Discussion}

\section{(1) Manganous Selenide}

(A) Plastic Deformation: The slip traces around Vickers indentations on the (001), (011), and (111) planes of $\mathrm{MnSe}$ revealed that the primary glide planes are $\{111\}$ (Fig. 1). The slip directions were assumed to be the close-packed directions on these planes, giving a Burgers vector $a / 2<1 \overline{1} 0\rangle$. On the (001) plane (Fig. 1(A)), the slip traces extended in $\langle 1 \overline{10}\rangle$ directions, whereas on the $(011)$ plane the traces were in [011] and $\langle 112\rangle$ directions (only those in the [0i1] direction are shown in Fig. 1(B) and unequivocally identified 
the slip planes as $\{111\})$. Slip traces on the (111) plane were also in $\langle 110\rangle$ directions, consistent with $\{111\}$ slip, but are not definitive since they could also arise from slip on either $\{100\}$ or $\{110\}$ planes.

Some $\{110\}<1 \overline{1} 0>$ slip was also observed as a secondary mechanism in samples mounted in Bakelite and quenched to $-70^{\circ} \mathrm{C}$ in an alcohol-dry ice solution. The differential thermal contraction between Bakelite and $\mathrm{MnSe}$ was sufficient to create a compressive stress in the MnSe great enough to cause $\{111\}$ slip and cross slip on $\{110\}$ planes. This could be avoided by using solder as a mounting material.

The $\{111\}<1 \overline{1} 0>$ primary slip mechanism of MnSe corresponds to that found in fcc metals and in the NaCl-type compounds UC ${ }^{8}$ and TiC. ${ }^{8}$ This is in contrast to the $\left.\{110\}<1 \overrightarrow{10}\right\rangle$ primary glide mechanism found in $\mathrm{MgO},{ }^{\circ} \mathrm{MnS}^{10}$ and $\mathrm{MnO}^{11}$ and, indeed, in most $\mathrm{NaCl}$-type materials where the bonding is largely ionic. This tendency in MnSe toward more metallic behavior is also reflected in other material properties such as color (darker), hardness (lower), electrical conductivity (higher), and anion polarizability (greater), and indicates that the chemical bond in MnSe is more covalent-metallic and less ionic than that in $\mathrm{MnS}$.

(B) Fracture: Manganous selenide shows primary and secondary cleavage on $\{100\}$ and $\{110\}$ planes, respectively. This behavior is similar to that of other materials with the NaCl-type structure. ${ }^{7}$

Surface indentation tended to produce primarily $\{100\}$ fracture as may be seen in Fig. 1(A). The cracks are concentrated near the corners of the impression in regions where perpendicular intersecting slip lines are also concentrated. It is quite possible that the $\{\mathbf{1 0 0}\}$ cracking is the result of a slip or dislocation interaction mechanism rather than simply a $\{100\}$ tension cleavage crack.

(C) Flow Around the Vickers Indenter: For certain orientations of the Vickers diamond pyramid relative to the surface of indentation, impressions of unusual shape were observed. Bückle $^{12}$ observed similarly shaped impressions in aluminum single crystals and concluded that the distortion was caused by a directional piling-up and sinking-in of material around the indenter.

Interference photomicrographs taken of Vickers impressions on the $\{001\}$ surface of $\mathrm{MnSe}$ reveal piled-up regions in $\langle 110\rangle$ directions and regions of sinking-in in $\langle 010\rangle$ directions. Figures $2(A)$ and $(B)$ show impressions in which the indenter diagonals were in $\langle 100\rangle$ and $\langle 110\rangle$ directions, indicating that this feature is independent of indenter orientation and is therefore a crystallographic effect. Thus, the indenter penetrating the surface causes crystallographic plastic flow leading to a pileup and sinking-in of material, the form of which depends on the symmetry of the slip systems of the crystal. The manner in which the indenter cuts these regions determines the shape of the impression.

A consequence of this deformation is that most of the slip traces were seen where the most extensive pileup occurred, and significantly fewer were visible elsewhere (Fig. 1(A)). The concentrations of slip lines and surface displacements in certain crystallographic directions are not unique to $\mathrm{MnSe}$, however. They were observed in $\mathrm{MgO}$ at high temperatures by Keh" and in the MnS used in this study, both of which have $\{110\}<1 \overline{1} 0\rangle$ as the primary glide mechanism. In $\mathrm{MgO}$ four "hills" of material in $\langle 1 \overline{1} 0\rangle$ directions around indentations on $\{001\}$ were observed. ${ }^{9}$ In $\mathrm{MnS}$ the pileup was analogous to that found in MnSe except that it was less extensive and there was no sinking-in.

As was previously mentioned, fracture around Vickers indentations in MnSe occurred predominantly on $\{100\}$ planes. Around indentations in $\mathrm{MgO}^{\circ}$ and $\mathrm{MnS}^{10}$ however, primarily $\{110\}$ fracture was evident. Keh ${ }^{9}$ attributed the $\{110\}$ fracture in $\mathrm{MgO}$ to an interaction of dislocations on intersecting $\{101\}$ planes, such as
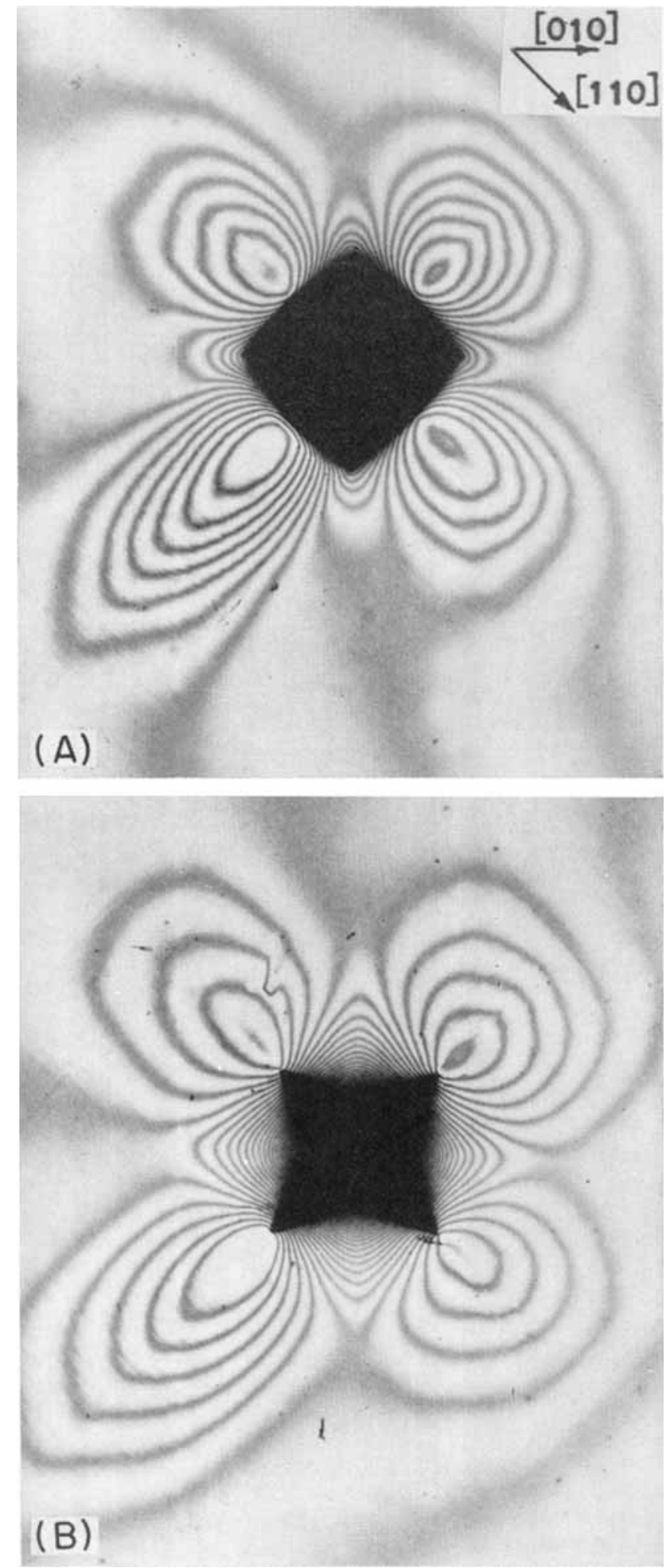

Fig. 2. Interference photomicrographs around Vickers hardness impressions on the (001) surface of an MnSe erystal with the indenter diagonals aligned parallel to the $(A)<010>$ and $(B)<110>$ directions $(\times 200)$.

$$
\mathbf{a} / 2[0 \overline{1} 1]+\mathbf{a} / 2[101] \rightarrow a / 2[110]
$$

resulting in an immobile edge dislocation lying on a (112) plane. Moving dislocations can pile up against this barrier and initiate a crack in the $(1 \overline{1} 0)$ plane. With sufficient thermal energy, portions of the piled-up dislocations could crossglide to other parallel glide planes, reducing the tendency for crack formation and leading to a material pileup like that cited earlier. A simpler way of viewing this is to note that 
slip on two adjacent $\{101\}$ planes tends to separate material along a third $\{110\}$ plane containing their line of intersection.

Similarly, the $\{100\}$ fracture in MnSe may be viewed as a consequence of the tendency for material to undergo additive slip in two $\langle 110\rangle$ directions on each $\{111\}$ plane (net $\langle 112\rangle$ flow on each plane) which would tend to separate material along a $\{100\}$ plane. It is also possible to postulate several energetically favorable dislocation interactions forming sessile dislocations leading to a dislocation pileup and, in the absence of sufficient cross slip, to eventual $\{100\}$ fracture. Two of these are:

$$
\begin{aligned}
& \mathbf{a} / 2[110]+\mathbf{a} / 2[\overline{1} 10] \rightarrow \mathbf{a} / 2[020]=\mathbf{a}[010] \\
& \mathbf{a} / 2[110]+\mathbf{a} / 2[011] \rightarrow \mathbf{a} / 2[10 \overline{1}]
\end{aligned}
$$

Both of the resulting dislocations are of the edge variety and lie along the [101] intersection of two adjacent $\{111\}$ planes. The first has a glide plane (101) and the second (010).

From the preceding discussion it is indicated that plastic deformation leading to a material pileup is quite intimately related to fracture caused by barriers to plastic flow. Where slip and cross-glide are easier (i.e. at higher temperatures or in MnSe versus $\mathrm{MnS}$ ) fracture is less and material pileup more pronounced.

\section{(2) MnSe-MnS System}

(A) Plastic Deformation: Although $\{110\}<110\rangle$ was observed as a secondary mechanism in MnSe, it was not detected as slip traces around Vickers indentations. With as little as 10 at.\% substitution of sulfide in selenide, however, traces due to $\{110\}<110>$ slip were detected. As the sulfide content increased, the amount of $\{110\}$ slip became more pronounced. Figure 3 shows these traces and a $\{100\}$ crack around a Vickers indentation on the (001) plane of a 50 at.\% MnSe-50 at.\% MnS sample. Those traces concentrated at the corners of a square are due to $\{110\}$ slip, whereas those in $\langle 110\rangle$ directions are from $\{111\}$ slip. The primary slip mechanisms were found to change gradually with composition, both mechanisms being operative for all intermediate compositions studied.

(B) Fracture: The fracture modes associated with surface indentation also change gradually with composition from primarily $\{100\}$ fracture for $\mathrm{MnSe}$ to $\{110\}$ for $\mathrm{MnS}$. As an example, Fig. 3 shows a $\{100\}$ crack in a sample of $50-50$ composition.

(C) Diamond Pyramid Hardness: Figure 4 shows the variation of diamond pyramid (Vickers) hardness with composition at $-70^{\circ} \mathrm{C}$, room temperature, and $135^{\circ} \mathrm{C}$. No slip mechanisms other than those discussed were found over this temperature range, and it was impossible to distinguish if significantly different amounts of $\{110\}<110\rangle$ or $\{111\}$ $\langle 1 \overline{1} 0\rangle$ glide took place with temperature variation by the observation of slip traces alone. Hardnesses on the (001), (011) and (111) planes were the same within the $\pm 4 \%$ limits of the experimental error.

Manganous selenide is softer than manganous sulfide (47 $\mathrm{kg} / \mathrm{mm}^{2}$ vs $145 \mathrm{~kg} / \mathrm{mm}^{2}$ at $20^{\circ} \mathrm{C}$ ), and its hardness is less sensitive to temperature. In addition, there is a large positive deviation from linearity (from a line between end-members) in the solid solution hardening which accompanies the substitution of sulfide for selenide ions. The substitution of selenide for sulfide ions also produces a positive deviation.

Satisfactory hardness results were obtained for $\mathrm{MnS}$ at liquid nitrogen temperature $\left(\mathrm{DPH}=208 \mathrm{~kg} / \mathrm{mm}^{2}\right)$, showing significant amounts of $\{111\}$ slip with extensive $\{110\}$ cracking. Apparently dislocation movements on $\{110\}<1 \overline{10}\rangle$ slip systems are sufficiently hindered at low temperatures so that, energetically, it now becomes just as easy to activate appreciable amounts of $\{111\}$ slip, the secondary mechanism at room temperature. It was not possible to obtain reliable hardnesses

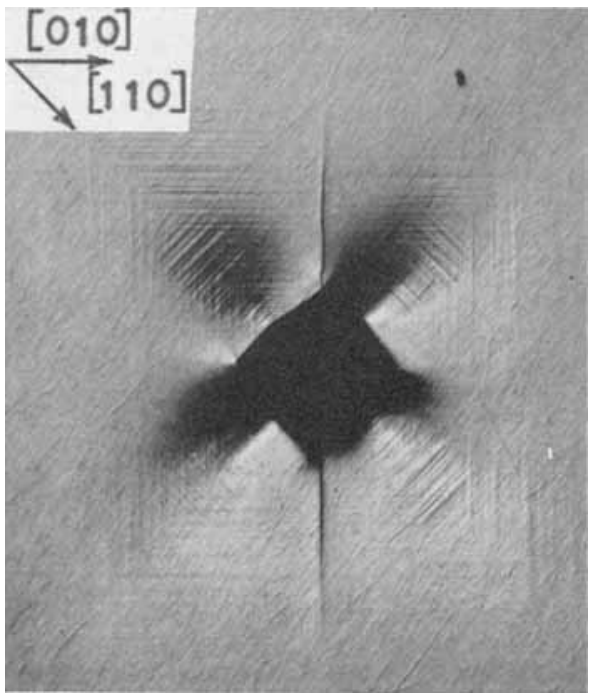

Fig. 3. Vickers hardness impression on the (001) surface of a 50 at. $\%$ MnSe-50 at. \% MnS crystal showing a $\{100\}$ crack and traces due to $\{111\}$ and $\{110\}$ slip $(\times 150)$.

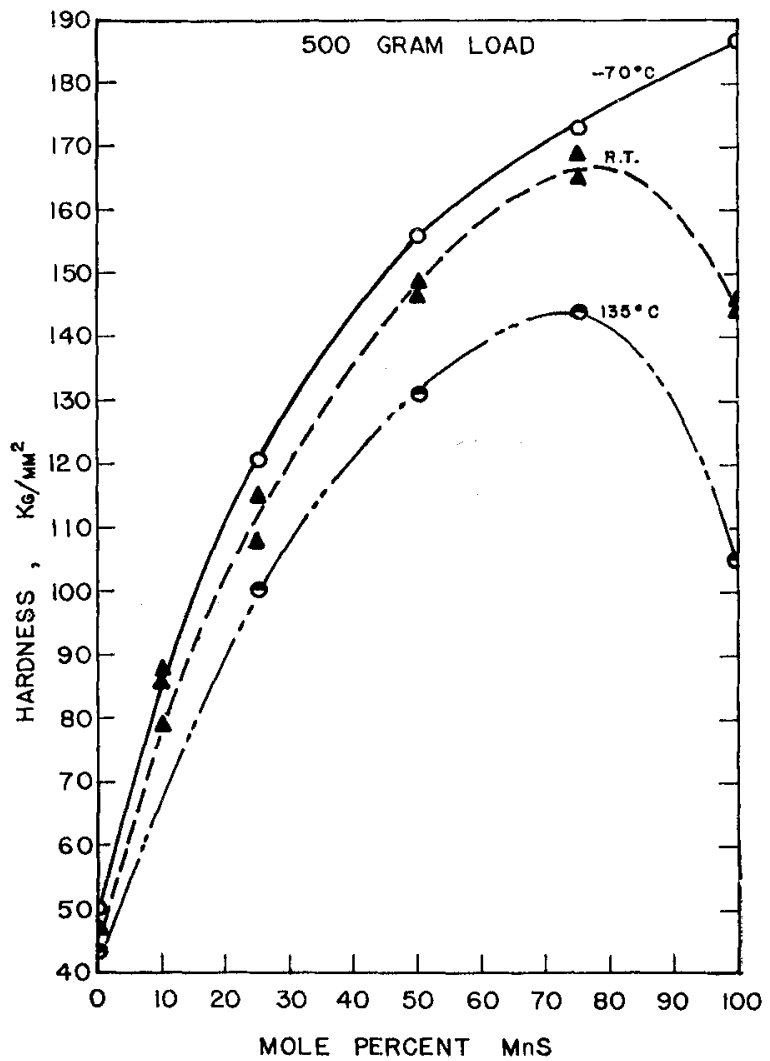

Fig. 4. Diamond pyramid hardnesses for MnSe-MnS solid solutions at $+135^{\circ} \mathrm{C}$, room temperature, and $-70^{\circ} \mathrm{C}$.

at liquid nitrogen temperature for samples containing $\mathrm{MnSe}$ because of the polymorphic phase change which occurs, ${ }^{4}$ as mentioned previously.

(D) Knoop Hardness: The variation of Knoop hardness with the orientation of the long axis of the indenter on anisotropic materials is a well-known phenomenon. On single crystal specimens, the hardness anisotropy is a complicated function of the particular slip systems of the crystal most favorably oriented to accommodate the shape change imposed by the indenter. Because of the difference in primary slip 
Table I. Knoop Hardness of MnSe

\begin{tabular}{ccccc}
\hline $\begin{array}{c}\text { Plane of } \\
\text { indentation }\end{array}$ & \multicolumn{4}{c}{ Direction of long axis of indenter } \\
\cline { 2 - 5 } & $<010\rangle$ & $\langle 011\rangle$ & $\langle 111\rangle$ & $\langle 112\rangle$ \\
\hline 100$\}$ & 65 & 43 & & \\
$\{11\}$ & 73 & 48 & 54 & 54 \\
$111\}$ & & 57 & & 57 \\
\hline
\end{tabular}

Table II. Knoop Hardness of MnS

\begin{tabular}{ccccc}
\hline $\begin{array}{c}\text { Plune of } \\
\text { indentation }\end{array}$ & \multicolumn{4}{c}{ Direction of long axis of indenter } \\
\cline { 2 - 5 } & $<010\rangle$ & $\langle 011\rangle$ & $\langle 11\rangle$ & $\langle 112\rangle$ \\
\hline$\{100\}$ & 122 & 142 & & \\
$\{011\}$ & 119 & 142 & 142 & 140 \\
$\{111\}$ & & 140 & & 140 \\
\hline
\end{tabular}

mechanism, this function is different for MnSe than for MnS. Room temperature Knoop hardness results for these materials are tabulated in Tables I and II which show that, for example, on the $\{100\}$ plane, MnSe is softer when the long axis of the indenter is parallel to $\langle 011\rangle$ than when it is aligned in $<010\rangle$, whereas the reverse is true for $\mathrm{MnS}$.

Because of the reversal of hard and soft directions for the two compounds, it becomes possible to follow the transition in primary glide mechanism by plotting the Knoop hardnesses for two indenter orientations. These results are shown in Fig. $5(A)$ for two orientations on the $(001)$ plane. Figure $5(B)$ indicates that similar tests at $-70^{\circ} \mathrm{C}$ showed a slight increase in the hardness of the selenide but a marked increase in the sulfide hardness and resulted in a shift of the crossover point of the two curves toward higher percentages of $\mathrm{MnS}$. This is a result of the relative temperature sensitivity of the $\{110\}$ $\langle 110\rangle$ and $\{111\}<110\rangle$ slip mechanisms. With decreased temperature, the $\{111\}<110\rangle$ mechanism contributes more to the overall deformation and, consequently, shifts the crossover to the right. Similarly, at higher temperatures, the crossover would be expected to shift to the left. This effect was observed.

\section{Conclusions}

1. Cubic manganous selenide (MnSe) of the NaCl-type deforms by $\{111\}<1 \overline{10}>$ and $\{110\}<1 \overline{10}>$ as primary and secondary slip mechanisms, respectively. This is in contrast to the $\{1.10\}<110>$ mechanism preferred by $\mathrm{MgO}$ and $\mathrm{MnS}$.

2. The primary cleavage planes in MnSe are $\{100\}$ with some secondary $\{110\}$ cleavage also evident. This is in agreement with $\mathrm{MgO}$ and $\mathrm{MnS}$; however, MnSe shows primarily $\{100\}$ fracture around surface indentations in contrast to $\{110\}$ fracture common in $\mathrm{MnS}$.

3. A crystallographic pileup of material occurs in certain regions around Vickers indentations on MnSe and MnS and a sinking-in of material in others. The pileup directions, which are independent of indenter orientation, produce impressions which are not square. This phenomenon is related to the flow of material on crystallographic slip systems. For an indentation on the $\{001\}$ plane of $\mathrm{MnSe}$, for instance, material prefers to flow in two $\langle 1 \overline{1} 0\rangle$ directions on each $\{111\}$ plane giving an apparent Burgers vector in $\langle 11 \overline{2}\rangle$ and resulting in four piled-up hills along $\langle 110\rangle$ directions on $\{001\}$. Slip traces are concentrated in the piled-up regions as a result of the preferential plastic deformation.

4. In the continuous solid solution system MnSe-MnS, the primary slip mechanism changes gradually from $\{111\}$ $<110>$ for $\mathrm{MnSe}$ to $\{110\}<1 \overline{10}>$ for $\mathrm{MnS}$.
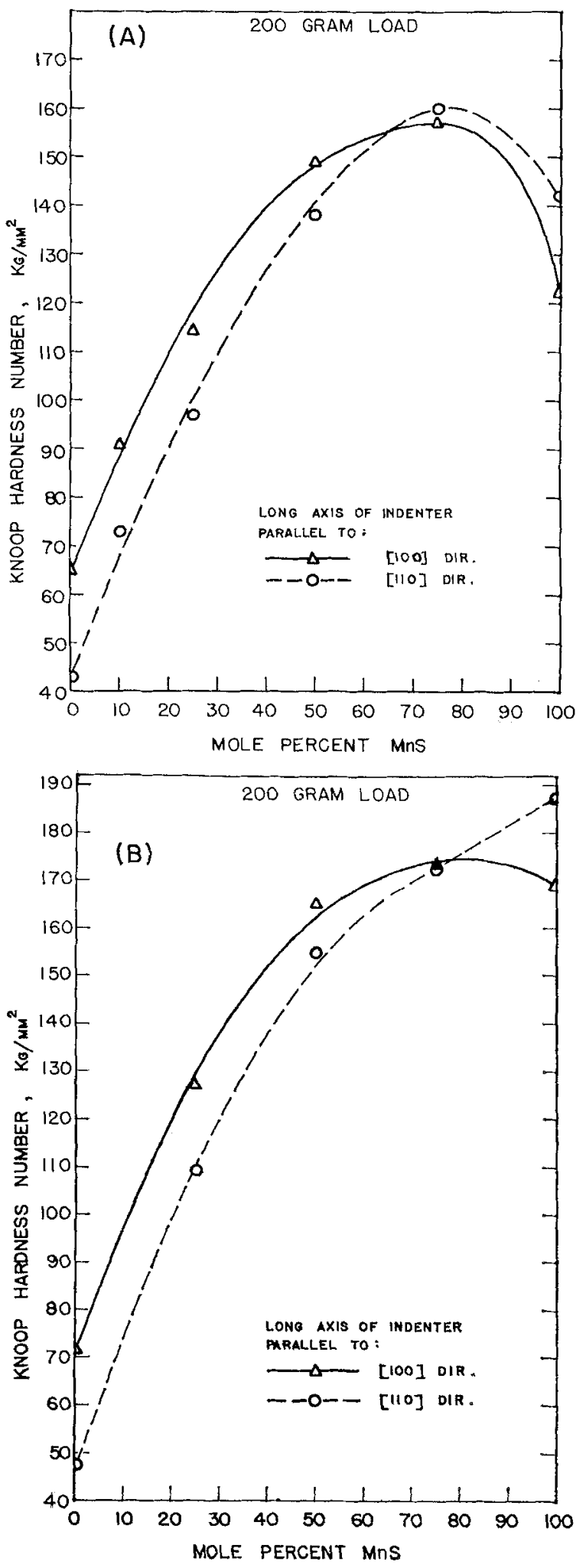

Fig. 5. Knoop hardness for two indenter orientations on the (001) surface for MnSe-MnS solid solutions at $(A)$ room temperature and $(B)-70^{\circ} \mathrm{C}$.

5. Substitution of sulfide for selenide ions in MnSe and of selenide for sulfide ions in $\mathrm{MnS}$ introduces a large positive solid solution-hardening deviation from simple linearity between the two end-member values. MnSe is much softer than MnS ( $47 \mathrm{~kg} / \mathrm{mm}^{2}$ vs $\left.145 \mathrm{~kg} / \mathrm{mm}^{2}\right)$. Within the limits of experimental error, the diamond pyramid hardness is the same on the $\{001\},\{011\}$, and $\{111\}$ planes in the MnSe-MnS 
system. The $\{110\}<1 \overline{1} 0>$ slip mechanism of $\mathrm{MnS}$ is more sensitive to decreasing temperature than the $\{111\}<1010\rangle$ mechanism. As a result $\mathrm{MnS}$ shows an appreciable amount of $\{111\}$ slip at liquid nitrogen temperature.

6. Knoop hardness anisotropy behavior is evident on the single crystal specimens. Manganese selenide is softest when the long axis of the Knoop indenter is in a $\langle 1 \overline{1} 0\rangle$ direction and hardest when it is in $\langle 100\rangle$. The reverse is true for MnS. The reversal of hard and soft directions in the MnSeMnS system provides a qualitative means of following the gradual change of primary glide mechanism with composition.

\section{Aeknowledgments}

The authors thank the Selenium-Tellurium Development Association for support in the reproduction of the original dissertation. Paul G. Riewald acknowledges the financial support he received as an NSF fellow.

Ref erences

${ }^{1}$ A. Taylor and B. J. Kagle, Crystallographic Data on Metal and Alloy Structures. Dover Publications, Inc., New York, 1963.

${ }^{1963 .}$ C. F. Squire, "Antiferromagnetism in Some Manganous Compounds," Phys. Rev., 56 [9] 922-25 (1939).
${ }^{3}$ H. P. Rooksby and N. C. Tombs, "Changes of Crystal Structure in Antiferromagnetic Compounds," Nature, 167 [4244] 364 (1951).

${ }^{4}$ N. N. Sirota and G. I. Makovetskii, "Neutron Diffraction Examination of MnSe," Dokl. Akad. Nauk Belorussk. SSR, 10 [8] 542-45 (1966)

J. M. Mehta, P. G. Riewald, and L. H. Van Vlack, "The System MnSe-MnS," J. Am. Ceram. Soc., 50 [3] 164 (1967).

B. Kiessling, B. Hassler, and C. Westman, "SelenideSulfide Inclusions and Synthetic Compounds of the (Mn, Me) (S, Se)-Type," J. Iron Steel Inst., 205 [5] 531-34 (1967).

${ }^{7} \mathrm{~J}$. J. Gilman; pp. 146-99 in Progress in Ceramic Science, Vol. 1. Edited by J. E. Burke. Pergamon Press, Inc., New York, 1961 .

${ }^{8}$ C. M. Van Der Walt and M. J. Sole, "Plastic Behavior of Crystals with the NaCl-Structure," Acta Mel., 15 [3] 459-62 (1967).

${ }^{\circ} \mathrm{A}$. S. Keh, "Dislocations in Indented Magnesium Oxide Crystals," J. Appl. Phys., 31 [9] 1538-45 (1960).

${ }^{10}$ H. C. Chao, L. Thomassen, and L. H. Van Vlack, "Deformation and Fracture of MnS Crystals," ASM Trans. Quart., 57 [2] 386-98 (1964).

${ }_{11}$ J "W Moore "Structure and Properties of Oriented Compound Eutectics"; Ph.D. Thesis, University of Michigan, 1965; Univ. Microfilms (Ann Arbor, Mich.), Order No. 66-6659, 169 pp.; Dissertation Abstr., 26 [12] 7243-44 (1966).

${ }^{12}$ H. Bückle, "Progress in Micro-Indentation Hardness Testing," Met. Rev., 4 [13] 49-100 (1959).

\title{
Mobility of Cation Vacancies in the Solid Solution KCl- $\mathrm{RbCl}$
}

\author{
J. B. HOLT,* H. G. SOCKEL, and H. SCHMALZRIED \\ Institut für Theoretische Hüttenkunde, Technische Universität Clausthal, Clausthal-Zellerfeld, Germany
}

\begin{abstract}
Of theoretical interest is the way in which the jump frequencies of cations $A^{+}$and $B^{+}$vary in the solid solution $\left(A^{+}, B^{+}\right) X^{-}$ as a function of composition. To provide experimental data for analysis, the electrical conductivity of various compositions of pure and doped $\mathrm{KCl}-\mathrm{RbCl}$ was measured, and from these measurements the cation vacancy mobility $\mu_{V\left(C^{+}\right)}$was calculated. In this system $\mu_{V\left(\mathrm{C}^{+}\right)}$was constant. The relation of this finding to mass transport properties is discussed.
\end{abstract}

\section{Introduction}

$N$ AN ionic crystal of pure $\mathrm{A}^{+} \mathrm{X}^{-}$at a defined temperature 1 where Schottky defects predominate, the electrochemical cation vacancy mobility $\mu_{V\left(A^{+}\right)}$has a certain value which is determined by the jump frequency between cations and cation vacancies. If $\mathrm{B}^{+} \mathrm{X}^{-}$is known to form a complete solid solution with $\mathrm{A}^{-1} \mathrm{X}$, the two compounds may be combined in such a way that the nearest neighbor cation environment surrounding any cation vacancy can systematically be changed from all $\mathrm{A}^{+}$to all $\mathrm{B}^{+}$ions. In this situation, at least three cases are worthy of consideration from a theoretical viewpoint.

Case I: The jump frequencies of cations $\mathrm{A}^{+}$and $\mathrm{B}^{+}$are the same and are independent of composition. This is the simplest of the three limiting cases and means that $\mu_{V\left(\mathrm{C}^{+}\right)}$is constant with composition ( $\mu_{V\left(C^{+}\right)}$represents the composite vacancy mobility due to both cations).

Case II: The jump frequencies of cations $\mathrm{A}^{+}$and $\mathrm{B}^{+}$are different and are independent of composition. This problem has been considered for dilute metallic solutions and can be solved under restricting assumptions, but a general solution has not yet been given.

Case III: The jump frequencies of cations $\mathrm{A}^{+}$and $\mathrm{B}^{+}$are different and depend on composition. This case is obviously still more complicated than case II.

Mobilities $\mu_{V\left(0^{+}\right)}$can easily be measured and the measured values should reflect the change in the average configuration of $\mathrm{A}^{+}$and $\mathrm{B}^{+}$cations around a given vacancy, as well as any variation in the jump frequency of the cations as the composition is varied from pure $\mathrm{A}^{+} \mathrm{X}^{-}$to pure $\mathrm{B}^{+} \mathrm{X}^{-}$. However, under the restrictions imposed by a solid solution, the cations $\mathrm{A}^{+}$and $\mathrm{B}^{+}$are expected to have similar physical characteristics. For this reason, variations of $\mu_{V\left(\mathrm{C}^{+}\right)}$with composition of the solid solution $\left(\mathrm{A}^{+}, \mathrm{B}^{+}\right) \mathrm{X}^{-}$may be quite small and thus difficult to measure experimentally.

Consider the compounds $\mathrm{RbCl}$ and $\mathrm{KCl}$, which are known to form a complete series of solid solutions. ${ }^{1}$ Since the electrical conductivity of pure and doped $\mathrm{KCl}$ has been thoroughly studied, ${ }^{2-5}$ the vacancy mobilities and concentrations are well known. On the other hand, few data are available in the literature on the electrical conductivity of $\mathrm{RbCl}$ and solid solution compositions of $\mathrm{RbCl}-\mathrm{KCl}$. Müller and Schmalzried in their investigation of the interdiffusion of $\mathrm{KCl}-\mathrm{RbCl}$ made sup-

Received November 18, 1968; revised copy received February 13, 1969 .

Supported in part by the United States Atomic Energy Commission.

* At the time this work was done, J. B. Holt was on sabbatical leave from Lawrence Radiation Laboratory, University of California, Livermore, California $94550 . \mathrm{He}$ was granted a fellowship by the Alexander von Humboldt Foundation in support of this work. 